\title{
LAS ORQUESTAS DE LOS COLISEOS MADRILEÑOS PRÍNCIPE Y CRUZ DURANTE LA SEGUNDA MITAD DEL SIGLO XVIII
}

\author{
The Orchestras of the Principe and Cruz Coliseums in \\ Madrid during the Second Half of the $18^{\text {th }}$ Century
}

\author{
Marina BARBA \\ Universidad Autónoma de Madrid
}

Fecha de recepción: 23/06/2015

Fecha de aceptación definitiva: 11/11/2015

RESUMEN: Durante la primera mitad del siglo XVIII no existieron plantillas orquestales estables en los teatros públicos madrileños, contratándose de manera puntual para los espectáculos que requiriesen un acompañamiento musical un número de intérpretes variable. En este trabajo se pretende dar a conocer el punto de inflexión de esta tendencia en la segunda mitad de la centuria y destacar la evolución, desarrollo y crecimiento continuo de los coliseos municipales, tanto en su repertorio como en sus infraestructuras. En base a la documentación conservada en la Sección de Secretaría del Archivo de Villa de Madrid, se estudiará en qué momento la orquesta es considerada un elemento regular, constante y necesario de las temporadas así como se trazará una línea de evolución referida al tipo de contratación de músicos para las compañías teatrales de los coliseos madrileños.

René Andioc y Mireille Coulon en su Cartelera teatral madrileña del siglo XVIII: (1708-1808) hacen referencia a las compañías que trabajaron en ambos teatros durante los últimos cincuenta años del siglo XVIII, las de Josef de Parra, María Hidalgo, José Martínez Gálvez, Juan Ángel, Águeda de la Calle, María Ladvenant, Nicolás de la Calle, Juan Ponce, Manuel Martínez, Eusebio Ribera, Joaquín Palomino, Luis Navarro y Francisco Ramos; mas sin abordar a los músicos que conformaban las orquestas. El primer trabajo en el que se trata este tema es el de José Máximo Leza 
LAS ORQUESTAS DE LOS COLISEOS MADRILEÑOS PRÍNCIPE Y CRUZ DURANTE LA SEGUNDA MITAD DEL SIGLO XVIII

"Las orquestas de ópera en Madrid entre los siglos XVIII-XIX", si bien no atañe a los coliseos Cruz y Príncipe durante la segunda mitad del siglo XVIII.

Palabras clave: Teatros urbanos de Madrid; orquestas; música; segunda mitad del siglo XVIII.

ABSTRACT: There were no stable orchestras in Madrid's public theatres during the first half of the Eighteenth Century, and a variable number of instrumentalists were used on each occasion. This paper discusses the development of the two townowned theatres in the second half of the century, when the orchestra was considered an important element and a new way of hiring musicians developed, based on sources kept at the Archivo de Villa de Madrid, Sección de Secretaría.

René Andioc and Mireille Coulon in their Cartelera teatral madrileña del siglo XVIII: (1708-1808) refer to the companies that worked in both theatres in the second half of the $18^{\text {th }}$ Century, those of de Josef de Parra, María Hidalgo, José Martínez Gálvez, Juan Ángel, Águeda de la Calle, María Ladvenant, Nicolás de la Calle, Juan Ponce, Manuel Martínez, Eusebio Ribera, Joaquín Palomino, Luis Navarro and Francisco Ramo, although they do not study the orchestral musicians. The first study on this issue is that by José Máximo Leza "Las orquestas de ópera en Madrid entre los siglos XVIII-XIX", although it does not discuss the theatres of La Cruz and El Príncipe in the second half of the century

Key words: Urban theaters in Madrid; Orchestras; Music; Second Half $18^{\text {th }}$ Century

La veleidad y volubilidad que caracterizan al teatro musical cortesano madrileño de la segunda mitad del siglo XVIII tras la llegada al trono de Carlos III ${ }^{1}$, contrasta con la existencia de una evolución, un desarrollo y un crecimiento continuo de los coliseos municipales, tanto en su repertorio como en sus infraestructuras. A partir de los documentos conservados en la Sección de Secretaría del Archivo de Villa de Madrid $^{2}$ relativos al funcionamiento de los teatros de la Cruz y del Príncipe desde 1750 a 1800 se van a definir los cinco diferentes segmentos de esta curva de evolución referida al tipo de contratación de músicos para las compañías teatrales de los coliseos madrileños:

1. Temporadas 1750-1751 a 1762-1763: músicos de compañía.

2. Temporadas 1763-1764 a 1766-1767: contratación puntual de agrupaciones orquestales para acompañar determinadas obras.

1. Carreras, Juan José. "Amores difíciles: la ópera de corte en la España del siglo XVIII». En CASAREs RODicio, Emilio y TORRente, Álvaro (eds.). La ópera en España e Hispanoamérica. Madrid: ICCMU, 2001, vol. I, pp. 205-230.

2. En adelante AVM, S. 
LAS ORQUESTAS DE LOS COLISEOS MADRILEÑOS PRÍNCIPE Y CRUZ DURANTE LA SEGUNDA MITAD DEL SIGLO XVIII

3. Temporadas 1767-1768 a 1772-1773: plantillas orquestales asociadas a las compañías para la representación de obras concretas.

4. Temporadas 1773-1774 a 1777-1778: plantillas orquestales asociadas a cada compañía para la temporada completa.

5. Temporadas 1778-1779 a 1799-1800: se retrocede al modelo anterior de contratación de orquestas asociadas a una compañía pero para funciones puntuales, no para toda la temporada teatral.

En el siguiente gráfico se pueden apreciar los diferentes segmentos de la curva de evolución que posteriormente se describirán de manera pormenorizada.

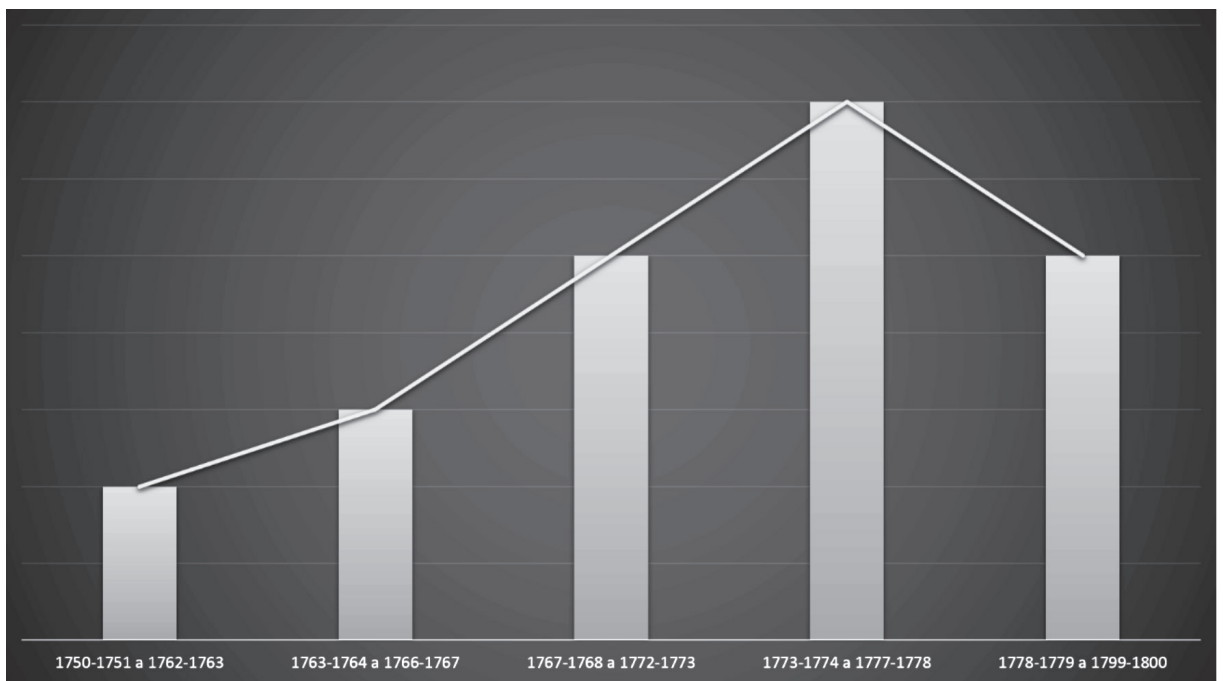

Figura 1. Segmentos de evolución de las orquestas por temporada

Durante las trece primeras temporadas teatrales correspondientes al primer segmento de la curva de evolución, se respeta el modelo de la primera mitad de la centuria: únicamente dos músicos formaban parte de la compañía y recibían un salario regular ${ }^{3}$; eran los denominados músicos de compañía encargados de realizar un acompañamiento para los espectáculos que así lo requiriesen ${ }^{4}$.

3. Leza, José Máximo. "Aspectos productivos de la ópera en los teatros públicos de Madrid (1730-1799)». En CASARES RODICIO, Emilio y TORRENTE, Álvaro (eds.). La ópera en España e Hispanoamérica. Madrid: ICCMU, 2001, vol. I, pp. 231-262.

4. Lolo, Begoña. "Itinerarios musicales en la tonadilla escénica». En Paisajes sonoros en el Madrid del s. XVIII: la tonadilla escénica. Madrid: Museo de San Isidro. Ayuntamiento de Madrid, 2003, p. 27. 
LAS ORQUESTAS DE LOS COLISEOS MADRILEÑOS PRÍNCIPE Y CRUZ DURANTE LA SEGUNDA MITAD DEL SIGLO XVIII

TABLA 1: TEMPORADAS 1750-1751 a 1762-1763

\begin{tabular}{|c|c|c|c|}
\hline AÑO & AUTOR & MÚSICO de COMPAÑÍA & $\mathrm{AVM}, \mathrm{S}$ \\
\hline $\begin{array}{l}1750-1751 \\
1751-1752\end{array}$ & Joseph Parra & $\begin{array}{l}\text { 1. }{ }^{\circ} \text { Manuel Francisco Ferreira } \\
2 .^{\circ} \text { Luis Antonio Rullet }\end{array}$ & $2 / 458 / 3$ \\
\hline \multirow[t]{2}{*}{$\begin{array}{l}1752-1753 \\
1753-1754\end{array}$} & Joseph Parra & $\begin{array}{l}\text { 1. }{ }^{\circ} \text { Manuel Francisco Ferreira } \\
\text { 2. }{ }^{\circ} \text { Luis Antonio Rullet }\end{array}$ & \multirow[t]{2}{*}{$\begin{array}{l}2 / 463 / 38 \\
2 / 458 / 25\end{array}$} \\
\hline & Manuel Guerrero & $\begin{array}{l}\text { 1. }{ }^{\circ} \text { Antonio Guerrero } \\
\text { 2. }{ }^{\circ} \text { Vicente Guerrero }\end{array}$ & \\
\hline \multirow[t]{2}{*}{$\begin{array}{l}1754-1755 \\
1758-1759\end{array}$} & Joseph Parra & $\begin{array}{l}\text { 1. }{ }^{\circ} \text { Manuel Francisco Ferreira } \\
\text { 2. }{ }^{\circ} \text { Luis Antonio Rullet }\end{array}$ & \multirow{2}{*}{$\begin{array}{l}2 / 458 / 28 \\
1 / 346 / 3 \\
1 / 367 / 1\end{array}$} \\
\hline & María Hidalgo & $\begin{array}{l}\text { 1. }{ }^{\circ} \text { Antonio Guerrero } \\
\text { 2. }{ }^{\circ} \text { Vicente Guerrero }\end{array}$ & \\
\hline \multirow[t]{2}{*}{$1759-1760$} & Joseph Parra & $\begin{array}{l}\text { 1. }{ }^{\circ} \text { Manuel Francisco Ferreira } \\
\text { 2. }{ }^{\circ} \text { Luis Antonio Rullet }\end{array}$ & \multirow[t]{2}{*}{$1 / 374 / 3$} \\
\hline & María Hidalgo & $\begin{array}{l}\text { 1. }{ }^{\circ} \text { Antonio Guerrero } \\
\text { 2. }{ }^{\circ} \text { Juan Manuel López }\end{array}$ & \\
\hline \multirow[t]{2}{*}{$\begin{array}{l}1760-1761 \\
1761-1762\end{array}$} & María Hidalgo & $\begin{array}{l}\text { 1. }{ }^{\circ} \text { Manuel Francisco Ferreira } \\
\text { 2. Juan Manuel López }\end{array}$ & \multirow[t]{2}{*}{$\begin{array}{l}1 / 365 / 2 \\
1 / 371 / 2\end{array}$} \\
\hline & Joseph Martínez Galbe & $\begin{array}{l}\text { 1. Antonio Guerrero } \\
2^{\circ} \text { Luis Antonio Rullet }\end{array}$ & \\
\hline \multirow[t]{2}{*}{$1762-1763$} & Águeda de la Calle & $\begin{array}{l}\text { 1. }{ }^{\circ} \text { Manuel Francisco Ferreira } \\
\text { 2. Juan Manuel López }\end{array}$ & \multirow[t]{2}{*}{$1 / 361 / 2$} \\
\hline & María Hidalgo & $\begin{array}{l}\text { 1. }{ }^{\circ} \text { Antonio Guerrero } \\
\text { 2. }{ }^{\circ} \text { Luis Antonio Rullet }\end{array}$ & \\
\hline
\end{tabular}

Como se aprecia en la Tabla 1, dentro de los músicos de compañía existía una jerarquía, ya que aparecen diferenciados en primer y segundo lugar y, aunque van trabajando para diferentes compañías, la de Joseph Parra, Manuel Guerrero, María Hidalgo, Joseph Martínez Galbe y Águeda de la Calle; solamente son cinco: Manuel Francisco Ferreira, Luis Antonio Rullet, Antonio Guerrero, Vicente Guerrero y Juan Manuel López.

A partir de la temporada 1763-1764 tan solo se cita a un músico por compañía. Antonio Guerrero y Manuel Francisco Ferreira van a ser los compositores de las compañías de María Hidalgo, María Ladvenant y Nicolás de la Calle. 
LAS ORQUESTAS DE LOS COLISEOS MADRILEÑOS PRÍNCIPE Y CRUZ DURANTE LA SEGUNDA MITAD DEL SIGLO XVIII

TABLA 2: TeMPORADAS 1763-1764 a 1766-1767

\begin{tabular}{|c|l|l|c|}
\hline AÑO & \multicolumn{1}{|c|}{ AUTOR } & \multicolumn{1}{|c|}{ MÚSICO de COMPAÑÍA } & AVM, S \\
\hline 1763-1764 & María Hidalgo & 1. ${ }^{\circ}$ Antonio Guerrero & $1 / 362 / 2$ \\
\cline { 2 - 3 } 1764-1765 & María Ladvenant & 1..$^{\circ}$ Manuel Francisco Ferreira & $1 / 363 / 2$ \\
\hline 1765-1766 & María Hidalgo & 1..$^{\circ}$ Antonio Guerrero & $1 / 364 / 2$ \\
\cline { 2 - 3 } $1766-1767$ & Nicolás de la Calle & 1..$^{\circ}$ Manuel Francisco Ferreira & $1 / 365 / 2$ \\
\hline
\end{tabular}

Durante estas cuatro temporadas que se han diferenciado en el segundo segmento de la curva, si bien aún no se relaciona una plantilla orquestal fija con cada compañía, sí que aparecen formaciones orquestales para la representación de determinadas obras. A continuación se presentan dos ejemplos de dos plantillas orquestales diferentes fechadas el cinco de febrero de 1765: una para la comedia de Antonio Pablo Fernández La mágica florentina, y otra para la zarzuela de Antonio o Egidio R. Duni ${ }^{5}$ El paje mudo

TABLA 3: LISTA DE MÚSICOS PARA LA REPRESENTACIÓN DE LA COMEDIA DE ANTONIO PABLO FERNÁNDEZ LA MÁGICA FLORENTINA

\begin{tabular}{|l|l|c|}
\hline INSTRUMENTO & \multicolumn{1}{|c|}{ MÚSICO } & SALARIO: reales/día \\
\hline Violines & Manuel Carreras & 30 \\
& Ramonet & 20 \\
& Enzzig & 20 \\
& Pedro Guerra & 20 \\
& Oucenit & 20 \\
\hline Contrabajo & Joseph Bobadilla & 30 \\
\hline Oboes & Francisco Comina & 20 \\
& Bautista & 20 \\
\hline Fagot & Tarradellas & 20 \\
\hline \multirow{2}{*}{ Trompas } & Nicolás & 30 \\
& Cayetano Canaut & 30 \\
\hline \multicolumn{2}{|l}{ GASTO TOTAL: 260 reales/día } \\
\hline
\end{tabular}

5. LEZA, José Máximo. «El mestizaje ilustrado: influencias francesas e italianas en el teatro musical madrileño (1760-1780)». Revista de Musicología, 2009, XXXII, n. 2, pp. 503-546, p. 513: Leza plantea la duda del compositor de la zarzuela El paje mudo "Antonio o Egidio R. Duni».

6. AVM, S 1/365/1. 
LAS ORQUESTAS DE LOS COLISEOS MADRILEÑOS PRÍNCIPE Y CRUZ DURANTE LA SEGUNDA MITAD DEL SIGLO XVIII

TABLA 4: LISTA DE MÚSICOS PARA LA REPRESENTACIÓN DE LA ZARZUELA DE ANTONIO O EGIDIO R. DUNI EL PAJE MUDO

\begin{tabular}{|l|l|c|}
\hline INSTRUMENTO & \multicolumn{1}{|c|}{ MÚSICO } & SALARIO: reales/día \\
\hline Violines & Salvador & 30 \\
& Mariano & 20 \\
& Josef García & 20 \\
& Antonio Cruz & 20 \\
& Francisco Marcolini & 20 \\
& Feiras & 20 \\
\hline Contrabajo & Tomé & 30 \\
& Amores & 30 \\
\hline Oboes & Ocampo & 20 \\
& Floril & 30 \\
\hline Trompas & Manuel Enríquez & 30 \\
& Pedro & \\
\hline GASTO TOTAL: & 290 reales/día & \\
\hline
\end{tabular}

Ambas orquestas presentan una instrumentación semejante: cinco o seis violines sin diferenciar entre primeros y segundos; ausencia de violas y violones; y oboes y trompas como representantes de la sección de viento. La plantilla de la comedia sí que incluye además un fagot, mientras que la peculiaridad de la plantilla de la zarzuela es que dobla los contrabajos. Estos y las trompas son los que más cobran igualándose al salario del primer violín.

El siguiente segmento de la curva de evolución comienza en la temporada 1767-1768. Según Jesús Rubio Jiménez en su obra El Conde de Aranda y el teatro:

[...] En 1767, aprovechando la muerte de María Ladvenant y Nicolás de la Calle, el Conde de Aranda transformó la rutina de las compañías que trabajaban únicamente en uno de los coliseos madrileños y las hizo alternar entre el Príncipe y la Cruz disponiendo dos periodos: de Pascua a verano y de septiembre a carnaval [... $]^{7}$.

Según la documentación consultada en el AVM, esta alternancia se viene observando desde 1750, dato que corroboran René Andioc y Mireille Coulon en su Cartelera teatral madrileña del siglo XVIII: (1708-1808) ${ }^{8}$. La novedad que sí que se aprecia desde la temporada 1767-1768 hasta la de 1772-1773 es que se cita a los componentes de las orquestas conformadas para representar funciones puntuales relacionándolos con las compañías teatrales. Según se puede leer en la siguiente Tabla 5, los músicos de las compañías siguen siendo los mismos que en las cuatro temporadas precedentes: Antonio Guerrero y Manuel Francisco Ferreira. Cabe destacar la presencia de una única compañía durante la temporada 1771-1772.

7. Rubio Jiménez, Jesús. El Conde de Aranda y el teatro. Zaragoza: Ibercaja, 1998, p. 179.

8. ANDIOC, René y Coulon, Mireille. Cartelera teatral madrileña del siglo XVIII: (1708-1808). Madrid: Fundación Universitaria Española, 2008. 
LAS ORQUESTAS DE LOS COLISEOS MADRILEÑOS PRÍNCIPE Y CRUZ DURANTE LA SEGUNDA MITAD DEL SIGLO XVIII

TABLA 5: TEMPORADA 1767-1768 a 1772-1773

\begin{tabular}{|c|l|l|l|}
\hline AÑO & \multicolumn{1}{|c|}{ AUTOR } & \multicolumn{1}{|c|}{ MÚSICO de COMPAÑÍA } & AVM, S \\
\hline $1767-1768$ & María Hidalgo & $1 .^{\circ}$ Antonio Guerrero & $1 / 347 / 2$ \\
\cline { 2 - 3 } $1770-1771$ & Juan Ponce & $1 .^{\circ}$ Manuel Francisco Ferreira & $1 / 348 / 2$ \\
\hline $1771-1772$ & Manuel Martínez & $1 .^{\circ}$ Antonio Guerrero & $1 / 353 / 2$ \\
\hline $1772-1773$ & Manuel Martínez & $1 .^{\circ}$ Antonio Guerrero & $1 / 353 / 2$ \\
\cline { 2 - 3 } & Eusebio Ribera & $1 .^{\circ}$ Manuel Francisco Ferreira & \\
\hline
\end{tabular}

La primera plantilla orquestal asociada a una compañía es la que se describe para la representación, en el teatro Príncipe, de la zarzuela en dos actos de Pablo Esteve, Los jardineros de Aranjuez, durante las navidades de 1768 con la compañía de María Hidalgo?:

Tabla 6: Lista de músicos de la compañía de María Hidalgo para la ZaRZuela de Esteve LOS JARDINEROS DE ARANJUEZ

\begin{tabular}{|l|l|c|}
\hline INSTRUMENTO & \multicolumn{1}{|c|}{ MÚSICO } & SALARIO: reales/día \\
\hline Violines & ${\text { Francisco Marcolini }{ }^{10}}$ & 30 \\
& Palomino & 20 \\
& Mariano & 20 \\
& Antonio Cruz & 20 \\
& Francisco Manchado (viola) & 20 \\
& Serrano & 20 \\
& Font & 20 \\
& Feiras & 20 \\
\hline Viola & Supernumerario & 4 \\
\hline Violón & Sierra & 20 \\
\hline Contrabajos & Andrés Julián & 30 \\
& Ribero y Canales (alternando) & 30 \\
\hline Oboes & Ocampo & 20 \\
& Lorenzo & 20 \\
\hline Trompas & Manuel Enríquez & 30 \\
& Pedro & 20 \\
\hline Clave & Antonio Muñiz & \\
\hline GASTO TOTAL: & 374 reales/día & \\
\hline
\end{tabular}

9. AVM, S $1 / 438 / 2$.

10. La negrita indica que el músico ha pertenecido a la orquesta de los teatros con anterioridad. 
Los salarios se mantienen y, al menos siete músicos, estaban en la plantilla orquestal de la zarzuela El paje mudo, representada durante la temporada 17641765, cuando también trabajaba la compañía de María Hidalgo con el músico Antonio Guerrero. En la orquesta sigue sin existir una diferencia entre primeros y segundos violines pero como novedad se añade un violón, un clave y se incorporan violas, aunque una de ellas se nombra en la cuerda de los violines y la otra es supernumerario, es decir, sin plaza fija.

Con fecha 1 de febrero de 1770, en la temporada siguiente, se encuentra la lista de músicos de la compañía de Juan Ponce para la representación en el coliseo de la Cruz de la comedia El hechizado por fuerza de Antonio Zamora ${ }^{11}$ :

TABla 7: Lista de músicos de la COMPAÑÍA DE JUAN PONCE PARA LA COMEDIA DE ANTONIO ZAMORA EL HECHIZADO POR FUERZA

\begin{tabular}{|l|l|c|}
\hline INSTRUMENTO & \multicolumn{1}{|c|}{ MÚSICO } & SALARIO: reales/día \\
\hline Violines & Manuel Carreras & 22 \\
& Pedro Guerra & 15 \\
& Antonio Cruz & 15 \\
& Mínguez & 15 \\
& Basset & 15 \\
\hline Contrabajo & Joseph Bobadilla & 22 \\
\hline Oboes & Francisco Comina & 15 \\
& Manuel Julián & 20 \\
\hline Fagot & Vicente Julián & 15 \\
\hline \multirow{2}{*}{ Trompas } & Nicolás & 20 \\
& Guillermo Martorell & 20 \\
\hline Clave & Eusebio Moya & 20 \\
\hline GASTO TOTAL: 215 reales/día \\
\hline
\end{tabular}

Exceptuando el violinista Antonio Cruz que aparece asimismo en la plantilla de la compañía de María Hidalgo para la representación de la zarzuela de Pablo Esteve, los otros cinco músicos que habían participado con anterioridad en las funciones de los coliseos madrileños lo habían hecho en la comedia La mágica florentina. Si bien en la temporada de 1764-1765, cuando tuvo lugar la representación de dicha función, el autor Juan Ponce no trabajaba aún junto a ninguna compañía, sí que lo hacía el músico Manuel Francisco Ferreira. Es decir, se van perfilando dos orquestas relacionadas con cada una de las compañías y su

11. AVM, S $1 / 369 / 2$ 
músico: la de las zarzuelas El paje mudo y Los jardineros de Aranjuez; y las de las comedias La mágica florentina y El hechizado por fuerza.

En la orquesta de esta comedia de Antonio Zamora El hechizado por fuerza, se observa una disminución de la plantilla y de los salarios con respecto a la de las obras anteriormente representadas. Preciso es destacar que el clavecinista asciende a cobrar como los instrumentistas favorecidos, primer violín, contrabajo y trompas, y que por alguna razón desconocida el segundo oboe recibe cinco reales más de lo que le correspondería.

Lamentablemente, correspondiéndose con la siguiente temporada y fechada el 16 de octubre de 1771, se localiza una lista de los músicos de la compañía de Manuel Martínez para la representación en el coliseo Príncipe de la zarzuela Las segadoras de Vallecas del maestro Antonio Rodríguez de $\mathrm{Hita}^{12}$ y con libreto de Ramón de la Cruz; en la que no se especifica el nombre de los músicos por lo que se imposibilita su relación con las agrupaciones anteriores ${ }^{13}$.

TABla 8: LisTa de los MÚSICOS DE LA COMPAÑía DE MANUEL MARTíNEZ PARA LA ZARZUELA DE ANTONio Rodríguez de Hita y RAMÓn de la CRUZ LAS SEGADORAS DE VALLECAS

\begin{tabular}{|l|c|}
\hline INSTRUMENTO & $\begin{array}{c}\text { SALARIO: } \\
\text { reales/día por músico }\end{array}$ \\
\hline Primer violín & 30 \\
\hline 4 Violines & 20 \\
\hline 2 Violas & 20 \\
\hline 1 Violón & 20 \\
\hline 2 Contrabajos & 30 \\
\hline 2 Oboes & 20 \\
\hline 2 Trompas & 30 \\
\hline 1 Clave & 20 \\
\hline GASTO TOTAL: 350 reales/día \\
\hline
\end{tabular}

Los salarios son los mismos que para las zarzuelas El paje mudo y Los jardineros de Aranjuez, y, aunque la plantilla es básicamente la misma, se reduce el número de violines y por lo tanto el gasto total.

Para la siguiente temporada teatral de 1772-1773 se describe una plantilla orquestal para cada compañía destinadas a tocar en tres obras dramáticas distintas.

12. ReCASENS, Albert. Las zarzuelas de Antonio Rodríguez de Hita (1722-1787): Contribución al estudio de la zarzuela madrileña hacia 1760-1777. Thèse de doctorat. Louvain: Université Catholique de Louvain, 2001.

13. AVM, S $1 / 369 / 2$ 
LAS ORQUESTAS DE LOS COLISEOS MADRILEÑOS PRÍNCIPE Y CRUZ DURANTE LA SEGUNDA MITAD DEL SIGLO XVIII

Ambas orquestas constan de la misma plantilla y presupuesto ${ }^{14}$. Asociada a la compañía de Eusebio Ribera se encuentra la lista de músicos para la representación en el coliseo de la Cruz el 4 de octubre de 1772 de la tragedia San Alberto, y para la representación el 4 de noviembre de 1772 de la comedia de Agustín Salazar y Torres Los juegos olimpicos.

TABla 9: LISTA DE MÚSICOS DE LA COMPAÑÍA DE EUSEBIO RIBERA PARA LA TRAGEDIA SAN ALBERTO Y LA COMEDIA LOS JUEGOS OLÍMPICOS

\begin{tabular}{|l|l|c|}
\hline INSTRUMENTO & \multicolumn{1}{|c|}{ MÚSICO } & SALARIO: reales/día \\
\hline Violines & Manuel Carreras & 30 \\
& Basset & 20 \\
& Serrano & 20 \\
& Enríquez & 20 \\
& Montefano & 20 \\
& Andrés Monjui & 20 \\
\hline Violón & Ramón Monzón & 20 \\
\hline Contrabajo & Andrés Julián & 30 \\
\hline Oboes & Vicente Julián ${ }^{15}$ & 20 \\
\hline Trompas & Manuel Enríquez & 30 \\
& Guillermo Martorell & 30 \\
\hline Clave & Eusebio Moya & 20 \\
\hline \multicolumn{2}{|l|}{ GASTO TOTAL: 280 reales/día } \\
\hline
\end{tabular}

De los nueve músicos de la orquesta de la compañía de Eusebio Ribera que habían tocado con anterioridad en alguna orquesta de los coliseos, seis de ellos lo habían hecho vinculados a las compañías donde Manuel Francisco Ferreira era el músico.

Asociada a la compañía de Manuel Martínez encontramos la lista para la representación de la segunda parte de la comedia El asombro de Jerez, Juana la Rabicortona:

14. AVM, S $1 / 353 / 2$.

15. Preciso subrayar que Vicente Julián, quién aparece en esta plantilla como oboe, en la orquesta de la función que se representa el 1 de febrero de 1770 El hechizado por fuerza, tocaba el fagot. 
LAS ORQUESTAS DE LOS COLISEOS MADRILEÑOS PRÍNCIPE Y CRUZ DURANTE LA SEGUNDA MITAD DEL SIGLO XVIII

TABla 10: Lista de músicos de la compañía de Manuel MarTínez Para la REPRESENTACión DE EL ASOMBRO DE JEREZ, JUANA LA RABICORTONA

\begin{tabular}{|l|l|c|}
\hline INSTRUMENTO & \multicolumn{1}{|c|}{ MÚSICO } & SALARIO: reales/día \\
\hline Violines & Bautista & 30 \\
& Antonio Cruz & 20 \\
& Antonio Jáuregui & 20 \\
& Francisco Manchado & 20 \\
& Juan Bala & 20 \\
& Josef García & 20 \\
\hline Violón & Sierra & 20 \\
\hline Contrabajo & Joseph Bobadilla & 30 \\
\hline Oboe & Francisco García & 20 \\
\hline Trompas & Nicolás & 30 \\
& Pedro & 30 \\
\hline Clave & Antonio Muñiz & 20 \\
\hline GASTO TOTAL: 280 reales/día \\
\hline
\end{tabular}

De esta plantilla, seis de los ocho músicos habituales en los teatros de la corte los encontramos en las orquestas vinculadas a la compañía de Manuel Martínez y su músico Antonio Guerrero. A partir de estos datos podemos confirmar que la tendencia a pertenecer a una u otra compañía se consolida mas permitiendo cierta permeabilidad.

Hasta el momento, aunque según avanza el siglo se va desarrollando una cierta estabilidad orquestal, aún no se ha descrito ninguna plantilla que no se hubiera conformado teniendo como objetivo la representación de una determinada obra. En las siguientes cuatro temporadas incluidas en el cuarto segmento de la curva de evolución, se sitúa el punto de inflexión de esta práctica.

TABLA 11: TEMPORADAS: 1773-1774 A 1777-1778

\begin{tabular}{|c|c|c|c|}
\hline AÑO & AUTOR & MÚSICO de COMPAÑíA & AVM, S \\
\hline \multirow[t]{2}{*}{$\begin{array}{l}1773-1774 \\
1774-1775\end{array}$} & Manuel Martínez & $\begin{array}{l}\text { 1. }{ }^{\circ} \text { Antonio Guerrero } \\
\text { Orquesta de compañía }\end{array}$ & \multirow[t]{2}{*}{$\begin{array}{l}1 / 373 / 1 \\
1 / 355 / 1\end{array}$} \\
\hline & Eusebio Ribera & 1. ${ }^{\circ}$ Manuel Francisco Ferreira & \\
\hline \multirow[t]{2}{*}{$1775-1776$} & Manuel Martínez & \multirow[t]{2}{*}{ Orquestas de compañía } & \multirow[t]{2}{*}{$1 / 357 / 1$} \\
\hline & Eusebio Ribera & & \\
\hline \multirow[t]{2}{*}{$1776-1777$} & Manuel Martínez & 1. ${ }^{\circ}$ Manuel Francisco Ferreira & \multirow[t]{2}{*}{$1 / 359 / 2$} \\
\hline & Eusebio Ribera & & \\
\hline \multirow[t]{2}{*}{$1777-1778$} & Manuel Martínez & $\begin{array}{l}\text { 1. }{ }^{\circ} \text { Manuel Francisco Ferreira } \\
\text { Orquesta de compañía }\end{array}$ & \multirow[t]{2}{*}{$1 / 370 / 2$} \\
\hline & Eusebio Ribera & $\begin{array}{l}\text { 1. }{ }^{\circ} \text { Blas de Laserna } \\
\text { Orquesta de compañía }\end{array}$ & \\
\hline
\end{tabular}


LAS ORQUESTAS DE LOS COLISEOS MADRILEÑOS PRÍNCIPE Y CRUZ DURANTE LA SEGUNDA MITAD DEL SIGLO XVIII

Según se aprecia en la Tabla 11, en la temporada de 1775-1776 no se añaden los nombres de los músicos dentro de los miembros de la compañía sino directamente la orquesta, y en la siguiente temporada de 1776-1777 se omite el nombre del músico de la compañía de Eusebio Ribera, plaza que al año siguiente sería ocupada por el maestro Blas de Laserna. La primera plantilla orquestal de la compañía de Manuel Martínez es para la temporada 1773-1774 ${ }^{16}$.

Tabla 12: Plantilla orQuestal de la COMPaÑía de Manuel Martínez PARA LA TEMPORADA $1773-1774$

\begin{tabular}{|l|l|c|}
\hline \multicolumn{1}{|c|}{ INSTRUMENTO } & \multicolumn{1}{|c|}{ MÚSICO } & SALARIO: reales/día \\
\hline Violines primeros & Antonio Cruz & 30 \\
& Antonio Jáuregui & 20 \\
& Montefano & 20 \\
\hline Violines segundos & Francisco Marcolini & 20 \\
& Juan Lledó & 20 \\
& Francisco Manchado & 20 \\
\hline Violón & Sierra & 20 \\
\hline Contrabajo & Joseph Bobadilla & 30 \\
\hline Oboe & Francisco García & 20 \\
& Lorenzo & 20 \\
\hline Trompas & Jerónimo Germán & 30 \\
& Cayetano Canaut & 30 \\
& Su hijo & 30 \\
\hline Clave & Mateo & 20 \\
\hline Alquiler de timbales & Antonio Muñiz & 4 \\
\hline GASTO TOTAL: 364 reales/día & \\
\hline
\end{tabular}

Por primera vez se dividen los violines en primeros y segundos, se amplía la plantilla de trompas a cuatro y, exceptuando estas, casi la totalidad de los músicos han sido contratados en temporadas anteriores y de ellos solamente dos no han pertenecido a la compañía de Manuel Martínez: Cayetano Canaut y Montefano. Aunque no se cita intérprete se destaca el alquiler de timbales. Es la primera vez a lo largo de estos 50 años en los que se reseña la plantilla orquestal vinculada a una compañía pero tan solo a la de Manuel Martínez, de la de Eusebio Ribera no se recoge información.

16. AVM, S $1 / 438 / 2$. 
LAS ORQUESTAS DE LOS COLISEOS MADRILEÑOS PRÍNCIPE Y CRUZ DURANTE LA SEGUNDA MITAD DEL SIGLO XVIII

Esta situación varía en la temporada siguiente recogiéndose de esta manera: "Instrumentos que se van a tocar en las dos compañías entre el año de 1775 y el siguiente de $1776{ }^{17}$.

TABla 13: COMPaÑ̃́a De MANuel MarTínez TEMPORADA 1775-1776

\begin{tabular}{|l|l|c|}
\hline \multicolumn{1}{|c|}{ INSTRUMENTO } & \multicolumn{1}{c|}{ MÚSICO } & SALARIO: reales/día \\
\hline Violines & Antonio Cruz & $22+1 / 2$ \\
& Antonio Jáuregui & 12 \\
& Montefano & 12 \\
& Francisco Marcolini & 12 \\
\hline Violines supernumerarios & Josef García & 12 \\
& Juan Lledó & 12 \\
\hline Violón (vacante) & Sierra* & 16 \\
\hline Contrabajo & Joseph Bobadilla & 12 \\
\hline Oboe & Francisco García & 16 \\
\hline Trompas & Cayetano Canaut & 16 \\
\hline Clave & Jerónimo Germán* & 20 \\
\hline Timbales & Antonio Muñiz & \\
\hline GASTO TOTAL: $174+1 / 2$ reales/día \\
\hline
\end{tabular}

Los músicos de la orquesta son los mismos que los de la temporada anterior para esta misma compañía de Manuel Martínez por lo que podemos corroborar la existencia de una estabilidad y un vínculo. La única excepción la conforman los timbales por ser un puesto eventual en la orquesta. Según una nota en el expediente, Sierra es suplente en el puesto de violón ya que carece de nombramiento, y Jerónimo Germán, trompa, pasó a la Capilla Real a principio de la temporada y, aunque aparece como músico de la compañía, su puesto lo ocuparía un suplente del que se desconoce el nombre.

En general en esta temporada hay un descenso de salarios. El único que mantiene sus honorarios es el clavecinista Antonio Muñiz. El sueldo del timbalero así como el alquiler del instrumento se desconoce.

17. AVM, S $1 / 357 / 1$. 
LAS ORQUESTAS DE LOS COLISEOS MADRILEÑOS PRÍNCIPE Y CRUZ DURANTE LA SEGUNDA MITAD DEL SIGLO XVIII

TABla 14: COMPAÑía DE EusEbio RiBERA TEMPORADA 1775-1776

\begin{tabular}{|l|l|c|}
\hline \multicolumn{1}{|c|}{ INSTRUMENTO } & \multicolumn{1}{c|}{ MÚSICO } & SALARIO: reales/día \\
\hline Violines & Oliver & $22+1 / 2$ \\
& Andrés Monjui & $15^{*}$ \\
& Juan Bala & 12 \\
& Josef León & 12 \\
\hline Violines supernumerarios & Nicolás Pastor & 12 \\
& Isidoro Boccolo & 12 \\
\hline Violón (vacante) & Canales* & 12 \\
\hline Contrabajo & Ramón Monroy & 16 \\
\hline Oboe & Pablo Vacarell & 12 \\
\hline Trompas & Guillermo Martorell & 16 \\
& Jerónimo Boca & 16 \\
\hline Clave & Eusebio Moya & 20 \\
\hline GASTO TOTAL: $177+1 / 2$ reales/día \\
\hline
\end{tabular}

La diferencia en el gasto total de la orquesta de esta compañía en relación con la de Manuel Martínez son los tres reales de aumento de los que se benefició el violinista Andrés Monjui, según se especifica en el expediente. Canales ocupa el puesto de violón pero sin plaza, como ocurría también en la compañía de Manuel Martínez. Los salarios son idénticos en ambas orquestas y la mayoría de los músicos de esta plantilla de la compañía de Eusebio Ribera no han participado en temporadas anteriores. De los cuatro que sí lo han hecho tres han sido miembros de las orquestas relacionadas con el autor Eusebio Ribera o con el músico de su compañía Manuel Francisco Ferreira.

Las listas de los componentes de las compañías pertenecientes a la siguiente temporada teatral de 1776-1777, no recogen la plantilla orquestal. De nuevo, las únicas referencias las encontramos en funciones puntuales realizadas durante el verano $^{18}$. Durante el estío de 1776, la compañía de Manuel Martínez puso en escena dos zarzuelas en el teatro Príncipe. La primera de ellas, La fontana del placer, con música de José Castel y libreto de Bruno Solo de Zaldívar, se estrenó el 22 de julio; y la segunda, La majestad en la aldea, versionada a partir del libreto

18. Cotarelo y Mori, Emilio. Historia de la zarzuela o sea el drama lírico en España, desde su origen a fines del siglo XIX. Madrid: ICCMU, 2000, p. 136. En 1768 el Conde de Aranda, presidente del Consejo de Castilla y encargado de importantes reformas en los espectáculos, dispuso que en los días no feriados del estío las compañías de los coliseos madrileños tenían permitido dar funciones nocturnas a su propio beneficio. Para que estas resultaran más atractivas, la música estaba siempre presente, solían reunirse ambas orquestas y autores como Ramón de la Cruz, alejándose de sus gustos literarios, escribían libretos para este género que el autor de estas líneas denomina zarzuela urbana. 
LAS ORQUESTAS DE LOS COLISEOS MADRILEÑOS PRÍNCIPE Y CRUZ DURANTE LA SEGUNDA MITAD DEL SIGLO XVIII

operístico $^{19}$, se representó el 2 de agosto. Para ambas funciones se contó con la siguiente orquesta ${ }^{20}$ :

TABla 15: LisTa DE MÚSICOS DE la COMPaÑía de Manuel MarTíneZ PARA LA FONTANA DEL PLACER Y LA MAJESTAD EN LA ALDEA

\begin{tabular}{|l|l|c|}
\hline \multicolumn{1}{|c|}{ INSTRUMENTO } & \multicolumn{1}{|c|}{ MÚSICO } & SALARIO: reales/día \\
\hline Violines & Antonio Cruz & 30 \\
& Montefano & 20 \\
& Antonio Jáuregui & 20 \\
& Francisco Marcolini & 20 \\
\hline Violines supernumerarios & Juan Lledó & 20 \\
& Manuel Carriles & 20 \\
\hline Violas & Juan Bala & 20 \\
& Nicolás Pastor & 20 \\
\hline Violón & García & 20 \\
\hline Contrabajos & Joseph Bobadilla & 30 \\
& Ramón Monroy & 30 \\
\hline Oboes & Francisco García & 20 \\
& Lorenzo & 20 \\
\hline Trompas & Cayetano Canaut & 30 \\
& Mateo & 30 \\
\hline Claves & Antonio Muñiz & 20 \\
& Eusebio Moya \\
\hline GASTO TOTAL: 390 reales/día \\
\hline
\end{tabular}

Esta orquesta tuvo por aumento la sección de violas, un contrabajo, un oboe y un clave. Exceptuando el violón y uno de los violines supernumerarios, ambos sin plaza fija, el resto de músicos provienen de las orquestas de ambas compañías de la temporada anterior.

A su vez, ese mismo verano en el teatro de la Cruz, el cinco de agosto, la compañía de Eusebio Ribera puso en escena la zarzuela burlesca El tío y la tía, con música de Antonio Rosales y libreto de Ramón de la Cruz. La plantilla orquestal fue $e^{21}$ :

19. Ibid., p. 135. SuBIRÁ, José. Variadas versiones de libretos operísticos. Madrid: CSIC, 1973, p. 38.

20. AVM, S $1 / 360 / 2$

21. Ibidem. 
LAS ORQUESTAS DE LOS COLISEOS MADRILEÑOS PRÍNCIPE Y CRUZ DURANTE LA SEGUNDA MITAD DEL SIGLO XVIII

TABla 16: Lista de Músicos de la COMPAÑía de EUSEBIO RiBERA PARA EL TÍO Y LA TÍA

\begin{tabular}{|l|l|c|}
\hline \multicolumn{1}{|c|}{ INSTRUMENTO } & \multicolumn{1}{c|}{ MÚSICO } & SALARIO: reales/día \\
\hline Violines & Montefano & 30 \\
& Eustasio León & 20 \\
& Juan Bala & 20 \\
& Manuel Carriles & 20 \\
\hline Violines supernumerarios & Nicolás Pastor & 20 \\
& Isidoro Boccolo & 20 \\
\hline Violón & Josef Nielfa & 20 \\
\hline Contrabajo & Ramón Monroy & 30 \\
\hline Oboes & Francisco García & 20 \\
& Pablo Vacarell & 20 \\
\hline Trompas & Guillermo Martorell & 30 \\
& Jerónimo Boca & 30 \\
\hline \multicolumn{2}{|l|}{ GASTO TOTAL: 300 reales/día } \\
\hline
\end{tabular}

Se aprecia que algunos violines también han tocado en la orquesta de la compañía de Manuel Martínez y que el puesto de violón sigue sin ser una plaza fija. Como la plantilla es menor también lo son sus gastos. Esta misma orquesta trabajó de nuevo junto a la compañía de Eusebio Ribera en el teatro Cruz en la representación de la obra de Cervantes El cerco de Numancia, el 4 de diciembre de ese mismo año de 1776; y añadiendo un bufón y un tamboril que cobraron cada uno 20 reales por día, el 22 de diciembre en El triunfo de la inocencia.

De nuevo en la temporada 1777-1778 encontramos una orquesta asignada a cada compañía ${ }^{22}$.

Tal y como se puede ver en la siguiente Tabla 17, la orquesta de la compañía de Eusebio Ribera se beneficia de un aumento de sueldo, la incorporación en la plantilla de un copiante y de tener como músico o maestro compositor a Blas de Laserna.

A continuación, en la Tabla 18, se observa que la orquesta de la compañía de Manuel Martínez para esta temporada también obtuvo un aumento de salarios, si bien, tendría que esperar al año siguiente para tener un maestro compositor de la talla de Blas de Laserna que sería Pablo Esteve, y no cuenta en la plantilla con la figura del copiante aunque sí con un clave del que no se especifica el nombre y además no se beneficia del aumento de sueldo ya que era de los que ya cobraban 20 reales la función.

22. AVM, S $1 / 370 / 2$. 
LAS ORQUESTAS DE LOS COLISEOS MADRILEÑOS PRÍNCIPE Y CRUZ DURANTE LA SEGUNDA MITAD DEL SIGLO XVIII

TABla 17: ORQuesta DE LA COMPaÑía DE EUSEBio Ribera

\begin{tabular}{|l|l|c|}
\hline \multicolumn{1}{|c|}{ INSTRUMENTO } & \multicolumn{1}{c|}{ MÚSICO } & SALARIO: reales/día \\
\hline Violines & Andrés Monjui & $22 \rightarrow 30$ \\
& Eustasio León & $12 \rightarrow 20$ \\
& Juan Bala & $12 \rightarrow 20$ \\
& Manuel Carriles & $12 \rightarrow 20$ \\
\hline Violines supernumerarios & Nicolás Pastor & $12 \rightarrow 20$ \\
& Isidoro Boccolo & $12 \rightarrow 20$ \\
\hline Violón & Josef Nielfa & $12 \rightarrow 20$ \\
\hline Contrabajo & Ramón Monroy & $18 \rightarrow 30$ \\
\hline Oboes & Francisco García & $12 \rightarrow 20$ \\
& Pablo Vacarell & $12 \rightarrow 20$ \\
\hline Trompas & Jerónimo Boca & $16 \rightarrow 30$ \\
& Guillermo Martorell & $16 \rightarrow 30$ \\
\hline Copiante & Eusebio Mora & 20 \\
\hline GASTO TOTAL: $188 \rightarrow 300$ reales/día \\
\hline
\end{tabular}

Tabla 18: Orquesta de la compañ̃áa de Manuel Martínez

\begin{tabular}{|l|l|c|}
\hline \multicolumn{1}{|c|}{ INSTRUMENTO } & \multicolumn{1}{c|}{ MÚSICO } & SALARIO: reales/día \\
\hline Violines & Javier Francisco Cruz & $22 \rightarrow 30$ \\
& Ramón Montoyano & $12 \rightarrow 20$ \\
& Antonio Jáuregui & $12 \rightarrow 20$ \\
& Francisco Marcolini & $12 \rightarrow 20$ \\
\hline Violines supernumerarios & Mateo Machado & $12 \rightarrow 20$ \\
& Juan Lledó & $12 \rightarrow 20$ \\
\hline Violón & Josef Alarcón & $12 \rightarrow 20$ \\
\hline Contrabajo & Joseph Bobadilla & $18 \rightarrow 30$ \\
\hline Oboes & Manuel García & $12 \rightarrow 20$ \\
& Ignacio Pérez & $12 \rightarrow 20$ \\
\hline Trompas & Pascual Ouberia & $16 \rightarrow 30$ \\
& Cayetano Canaut & $16 \rightarrow 30$ \\
\hline Clave & \multicolumn{2}{|l}{20} \\
\hline GASTO TOTAL: $188+1 / 2 \rightarrow 300$ reales/día \\
\hline
\end{tabular}

A partir de la temporada de 1778-1779 y hasta el final del siglo, último segmento de la curva de evolución, no se vuelve a encontrar en la documentación del AVM ninguna orquesta vinculada a una compañía teatral. Tan solo se conservan de nuevo plantillas instrumentales requeridas para la representación de obras 
LAS ORQUESTAS DE LOS COLISEOS MADRILEÑOS PRÍNCIPE Y CRUZ DURANTE LA SEGUNDA MITAD DEL SIGLO XVIII

puntuales como la del siguiente ejemplo, interpretada por ambas orquestas, con inclusión de clarines y timbales, y donde se puede observar una anotación con el visto bueno de Blas de Laserna:

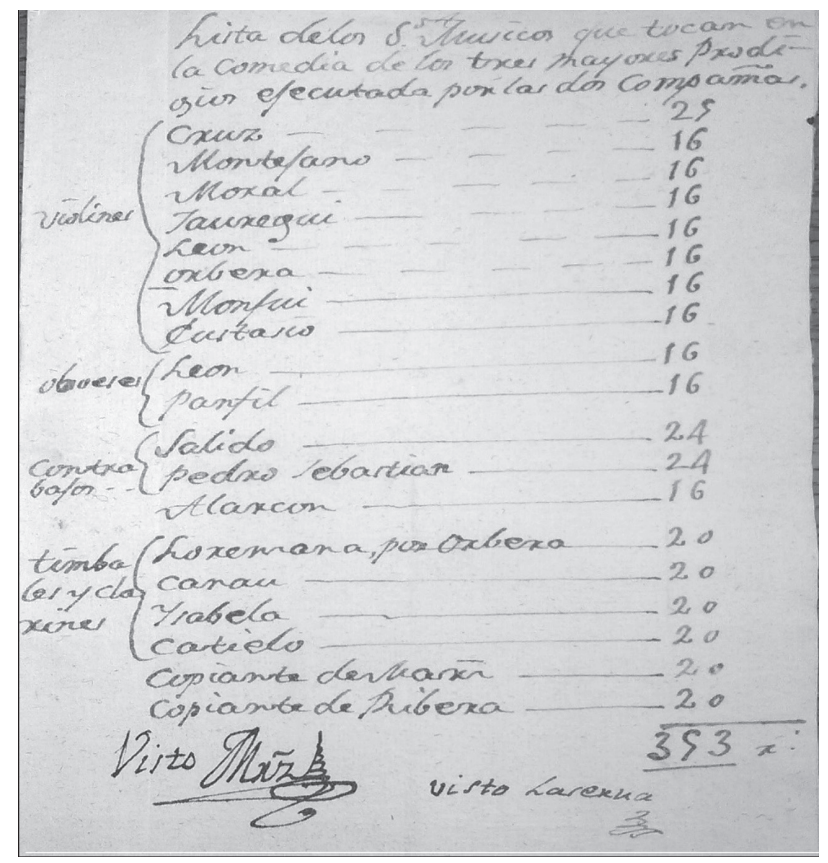

Figura 2. Orquesta de verano de 1793

La siguiente Tabla 19 contiene los nombres de los autores y músicos de compañía desde la temporada 1778-1779 hasta la de 1799-1800.

TABLA 19: TEMPORADAS 1778-1779 a 1799-1800

\begin{tabular}{|c|c|c|c|}
\hline AÑO & AUTOR & MÚSICO de COMPAÑÍA & AVM, S \\
\hline \multirow[t]{2}{*}{$1778-1779$} & Manuel Martínez & $\begin{array}{l}\text { 1. }{ }^{\circ} \text { Manuel Francisco Ferreira } \\
\text { Compositor Pablo Esteve }\end{array}$ & \multirow[t]{2}{*}{$1 / 372 / 2$} \\
\hline & Eusebio Ribera & 1. ${ }^{\circ}$ Blas de Laserna & \\
\hline \multirow[t]{2}{*}{$1779-1780$} & Manuel Martínez & $\begin{array}{l}\text { 1. }{ }^{\circ} \text { Manuel Francisco Ferreira } \\
\text { Compositor Pablo Esteve }\end{array}$ & \multirow[t]{2}{*}{$\begin{array}{l}1 / 374 / 2 \\
1 / 376 / 2\end{array}$} \\
\hline & Juan Ponce & & \\
\hline
\end{tabular}


LAS ORQUESTAS DE LOS COLISEOS MADRILEÑOS PRÍNCIPE Y CRUZ DURANTE LA SEGUNDA MITAD DEL SIGLO XVIII

\begin{tabular}{|c|c|c|c|}
\hline AÑO & AUTOR & MÚSICO de COMPAÑíA & AVM, S \\
\hline \multirow[t]{2}{*}{ 1780-1781 } & Manuel Martínez & $\begin{array}{l}\text { 1. }{ }^{\circ} \text { Francisco Méndez } \\
\text { Compositor Pablo Esteve }\end{array}$ & \multirow[t]{2}{*}{$1 / 377 / 2$} \\
\hline & Juan Ponce & 1. ${ }^{\circ}$ Fernando Fernandieri & \\
\hline \multirow[t]{2}{*}{ 1781-1782 } & Manuel Martínez & $\begin{array}{l}\text { 1. }{ }^{\circ} \text { Francisco Méndez } \\
\text { Compositor Pablo Esteve }\end{array}$ & \multirow[t]{2}{*}{$1 / 378 / 2$} \\
\hline & Joaquín Palomino & Compositor Blas de Laserna & \\
\hline \multirow[t]{2}{*}{$1782-1783$} & Manuel Martínez & $\begin{array}{l}\text { 1. }{ }^{\circ} \text { Francisco Méndez } \\
\text { Compositor Pablo Esteve }\end{array}$ & \multirow[t]{2}{*}{$1 / 380 / 2$} \\
\hline & Juan Ponce & Compositor Blas de Laserna & \\
\hline \multirow{2}{*}{$\begin{array}{c}1783-1784 \\
a \\
1790-1791\end{array}$} & Eusebio Ribera & Compositor Blas de Laserna & \multirow[b]{2}{*}{$\begin{array}{l}1 / 382 / 1 \\
1 / 384 / 1 \\
1 / 386 / 2 \\
1 / 388 / 2 \\
1 / 390 / 2 \\
1 / 393 / 2 \\
1 / 392 / 1 \\
1 / 399 / 1\end{array}$} \\
\hline & Manuel Martínez & Compositor Pablo Esteve & \\
\hline \multirow[t]{2}{*}{ 1791-1792 } & Eusebio Ribera & & \multirow[t]{2}{*}{$1 / 396 / 1$} \\
\hline & Manuel Martínez & Compositor Pablo del Moral & \\
\hline \multirow[t]{2}{*}{$1792-1793$} & Eusebio Ribera & Compositor Blas de Laserna & \multirow[t]{2}{*}{$1 / 397 / 2$} \\
\hline & Manuel Martínez & Compositor Pablo del Moral & \\
\hline \multirow{2}{*}{$\begin{array}{l}1793-1794 \\
1794-1795\end{array}$} & Eusebio Ribera & \multirow[t]{2}{*}{ Compositor Blas de Laserna } & \multirow{2}{*}{$\begin{array}{l}1 / 399 / 2 \\
1 / 400 / 3 \\
1 / 401 / 2\end{array}$} \\
\hline & Manuel Martínez & & \\
\hline \multirow[t]{2}{*}{$1795-1796$} & Francisco Ramos & & \multirow[t]{2}{*}{$1 / 404 / 4$} \\
\hline & Luis Navarro & & \\
\hline \multirow{2}{*}{$\begin{array}{l}1796-1797 \\
1797-1798\end{array}$} & Francisco Ramos & \multirow[t]{2}{*}{ Compositor Blas de Laserna } & \multirow{2}{*}{$\begin{array}{l}1 / 405 / 2 \\
1 / 407 / 2\end{array}$} \\
\hline & Luis Navarro & & \\
\hline \multirow[t]{2}{*}{ 1798-1799 } & Francisco Ramos & \multirow[t]{2}{*}{ Compositor Pablo del Moral } & \multirow[t]{2}{*}{$1 / 409 / 1$} \\
\hline & Luis Navarro & & \\
\hline \multirow[t]{2}{*}{ 1799-1800 } & Francisco Ramos & & \multirow[t]{2}{*}{$1 / 411 / 2$} \\
\hline & Luis Navarro & & \\
\hline
\end{tabular}

A lo largo de estos años cabe destacar algunas variaciones respecto a lo observado a lo largo de la segunda mitad de la centuria. En la temporada de 1778-1779 es la primera vez que se utiliza el término «compositor» para denominar 
LAS ORQUESTAS DE LOS COLISEOS MADRILEÑOS PRÍNCIPE Y CRUZ DURANTE LA SEGUNDA MITAD DEL SIGLO XVIII

el cargo de Pablo Esteve en la compañía de Manuel Martínez; mientras que a Blas de Laserna se le continúa llamando «músico» ${ }^{23}$. Habrá que esperar a la temporada de 1781-1782 para que ambos sean acreditados con la denominación de compositor $^{24}$, con un salario de 15 reales por día, igual que la primera dama o el primer galán de la compañía, mientras que el músico cobra 10 reales y medio por función, un salario medio dentro de la plantilla ${ }^{25}$.

En 1780 aparece un nuevo músico en la compañía de Manuel Martínez, Francisco Méndez, que sustituye a Manuel Francisco Ferreira y que tan solo cobra 8 reales y medio por función hasta que en la temporada de 1784 le aumentan a 10 reales; y en la compañía de Juan Ponce se incluye a otro músico nuevo, Fernando Fernandieri, que cobra 9 reales por función. En el puesto de músico dentro de la compañía de Manuel Martínez, se cita a Francisco Méndez en las temporadas 1781-82, 1782-83, 1784-85, 1787-88, 1788-89.

En 1791 aparece por primera vez como compositor de la compañía de Manuel Martínez, Pablo del Moral que sustituye a Pablo Esteve, quien moriría tres años después, en 1794.

Según el comportamiento observado a lo largo de la segunda mitad del siglo XVIII en las orquestas de los coliseos madrileños Príncipe y Cruz, se puede concluir que los músicos mantenían una estabilidad concreta, utilizando este término en el sentido de no continua, con las compañías teatrales. Aunque en una gran mayoría los instrumentistas aparecen vinculados al trabajo de los coliseos, tan solo durante cuatro temporadas están contratados de forma ininterrumpida como la orquesta de la compañía.

\section{BIBLIOGRAFÍA}

ACKer, Yolanda. Música y danza en el Diario de Madrid. Noticias, avisos y artículos (17581808). Madrid: Centro de Documentación de Música y Danza, 2007.

ANDIOC, René. Teatro y sociedad en el Madrid del siglo XVIII. Madrid: Castalia, 1987.

ANDIOC, René y COUlON, Mireille. Cartelera teatral madrileña del siglo XVIII: (17081808). Madrid: Fundación Universitaria Española, 2008.

Carmena y Millán, Luis. Crónica de la ópera italiana en Madrid desde el año 1738 hasta nuestros dias. Madrid: ICCMU, 2002.

CARreras, Juan José. "Amores difíciles: la ópera de corte en la España del siglo XVIII». En CASARES ROdicio, Emilio y TORRENTE, Álvaro (eds.). La ópera en España e Hispanoamérica. 2 Vols. Madrid: ICCMU, 2001, vol. 1, pp. 205-230.

CARreras, Juan José. «L'opera di corte a Madrid (1700-1759)». En Bellina, Anna Laura (ed.). Il teatro dei due mondi. L'operaitaliana nei paesi di lingua iberica. Treviso: Diastema Libri, 2000, pp. 11-35.

23. AVM, S $1 / 372 / 2$

24. AVM, S $1 / 378 / 2$

25. AVM, S 1/374/2 AVM, S 1/376/2. 
LAS ORQUESTAS DE LOS COLISEOS MADRILEÑOS PRÍNCIPE Y CRUZ DURANTE LA SEGUNDA MITAD DEL SIGLO XVIII

Cotarelo y Mori, Emilio. Historia de la zarzuela o sea el drama lírico en España, desde su origen a fines del siglo XIX. Madrid: ICCMU, 2000.

COTARElo y MORI, Emilio. Orígenes y establecimiento de la ópera es España hasta 1800. Madrid: ICCMU, 2004.

LABrador, Germán. "Música, Poder e Institución: La Real Capilla de Carlos IV (1788-1808)». Revista de Musicología, 2003, XXVI, n. ${ }^{\circ}$ 1, pp. 233-266.

LezA, José Máximo. "Aspectos productivos de la ópera en los teatros públicos de Madrid (1730-1799)». En CASARES RODICIO, Emilio y TORRENTE, Álvaro (eds.). La ópera en España e Hispanoamérica. 2 vols. Madrid: ICCMU, 2001, vol. 1, pp. 231-262.

LEZA, José Máximo. "El mestizaje ilustrado: influencias francesas e italianas en el teatro musical madrileño (1760-1780)». Revista de Musicología, 2009, XXXII, n. ${ }^{\circ}$ 2, pp. 503-546.

LEZA, José Máximo. "Las orquestas de ópera en Madrid entre los siglos XVIII-XIX». En Lolo, Begoña (ed.). Campos interdisciplinares de la musicología: V Congreso de la Sociedad Española de Musicología (Barcelona, 25-28 de octubre de 2000). 2 vols. Madrid: Sociedad Española de Musicología, 2002, vol. 1, pp. 115-139.

LoLO, Begoña. "Itinerarios musicales en la tonadilla escénica». En Paisajes sonoros en el Madrid del s. XVIII: la tonadilla escénica. Madrid: Museo de San Isidro. Ayuntamiento de Madrid, 2003.

Palacios, Emilio. El teatro popular español del siglo XVIII. Lleida: Milenio, 1998.

RECASENS, Albert. Las zarzuelas de Antonio Rodríguez de Hita (1722-1787): Contribución al estudio de la zarzuela madrileña hacia 1760-1777. Thèse de doctorat. Louvain: Université Catholique de Louvain, 2001.

Rubio Jiménez, Jesús. El Conde de Aranda y el teatro. Zaragoza: Ibercaja, 1998.

SUBIRÁ, José. Variadas versiones de libretos operísticos. Madrid: CSIC, 1973.

SUBIRÁ, José. Estudios sobre el teatro madrileño. La participación eventual de instrumentos no orquestales en la tonadilla. Madrid: Sección de Cultura e Información, Artes Gráficas Municipales, 1947.

SuBIRÁ, José. Historia de la música teatral en España. Madrid: Labor, 1945.

Subirá, José. La tonadilla escénica. 3 vols. Madrid: Real Academia Española, 1928-1930.

SuBIRÁ, José. "Los conciertos espirituales del s. XVIII». En Temas musicales madrileños. Madrid: Instituto de Estudios Madrileños, 1971, pp. 15-24. 